\title{
First and second type self-similar solutions of implosions and explosions containing ultrarelativistic shocks
}

\author{
Re'em Sari \\ Theoretical Astrophysics 130-33, California Institute of Technology, Pasadena, California 91125
}

(Received 27 December 2005; accepted 12 January 2006; published online 24 February 2006)

\begin{abstract}
We derive self-similar solutions for ultrarelativistic shock waves propagating into cold material of power law density profile in radius $\rho \propto r^{-k}$. We treat both implosions and explosions in three geometries: planar, cylindrical, and spherical. For spherical explosions these are the first type solutions of Blandford and McKee for $k<4$; they are the second type solutions found by Best and Sari for $k>5-\sqrt{3 / 4}$. In addition we find new, hollow (with evacuated interior), first type solutions that may be applicable for $4<k<17 / 4$. This "sequence" with increasing $k$ of first type solutions, hollow first type solutions, and then second type solutions is reminiscent of the nonrelativistic sequence. However, although in the nonrelativistic case there is a range of $k$ which corresponds to a "gap"- a range in $k$ with neither first nor second type solution which separates the hollow first type solutions and the second type solutions, here there is an "overlap": a range of $k$ for which current considerations allow for both hollow first and second type solutions. Further understanding is needed to determine which of the two solutions apply in this overlap regime. We provide similar exploration for the other geometries and for imploding configurations. Interestingly, we find a gap for imploding spherical shocks and exploding planar shocks and an overlap for imploding planar solutions. Cylindrical configurations have no hollow solutions and exhibit a direct transition from first type to second type solutions, without a gap or an overlap region. (C) 2006 American Institute of Physics. [DOI: 10.1063/1.2174567]
\end{abstract}

\section{INTRODUCTION}

Self-similar solutions provide some of the greatest simplifications to one-dimensional flows. Self-similarity allows the reduction of the partial differential equations, which contain two independent variables (space and time), into a set of ordinary differential equations (ODEs), where the single independent variable is a combination of space and time. The ODEs are then relatively easy to solve numerically or even analytically in some cases. They describe the asymptotic behavior of one-dimensional flows in a variety of circumstances, typically far away from the initial conditions and provided that the boundary conditions contain no spatial scale. (Some exceptions apply. For example, self-similarity can prevail in exponential density gradient in planar geometry.)

Perhaps the most famous hydrodynamic self-similar solution is that due to Taylor, ${ }^{1}$ Von Neumann, ${ }^{2}$ and Sedov, ${ }^{3}$ known as the Sedov-Taylor solution. It describes an explosion in which a strong shock wave propagates into cold surroundings whose density profile decreases as $\rho \propto r^{-k}$ (in the nonrelativistic literature, the density power law index is usually denoted by $\omega$ whereas in the relativistic literature the letter $k$ is used. Here we use the letter $k$ for both, as our focus is the relativistic regime). They used conservation of energy to obtain the scaling of the shock radius as a function of time. Such solutions are called first type solutions. Guderley ${ }^{4}$ (also see the discussion in Zeldovich and Raizer ${ }^{5}$ ) found a self-similar solution describing imploding shock waves in a constant density environment. In contrast to the strong explosion problem, energy considerations cannot be used to deduce the scaling of the shock radius as a function of time. Instead the scaling of the radius as a function of time must be found by demanding that the solution pass through a singular point of the equation. Such solutions are called self-similar solutions of the second type. More recently, Waxman and Shvarts ${ }^{6}$ showed that if the density falls fast enough $(k>3)$, energy considerations give the wrong scaling. They also showed that the solution should be of the second type for $k$ $>3.26$.

Recent astrophysical discoveries, mostly the afterglows of gamma ray bursts, led to an increased interest in the relativistic analogs of these solutions. We find that there is a considerable similarity between the relativistic and the nonrelativistic regimes. The relativistic version of the SedovTaylor solution was found by Blandford and McKee in 1976 (hereafter referred to as $\mathrm{BM}^{7}$ ). They provide the solution to the ultrarelativistic strong explosion problem in spherical geometry for density profiles with $k<4$. Best and $\mathrm{Sari}^{8}$ have found solutions of the second type for $k>5-\sqrt{3 / 4}$. Perna and Vietri $^{9}$ found relativistic solutions for shock waves propagating in an exponential density gradient. These are the relativistic analogs of the nonrelativistic solutions first found by Raizer. ${ }^{10}$ As this work was prepared, self-similar imploding relativistic shock waves for spherical geometry with constant density where numerically treated by Hidalgo and Mendoza. ${ }^{11}$

In this article, we provide a more complete exploration of the possible self-similar solutions in the ultrarelativistic case. We provide solutions in three geometries: planar, cylindrical, and spherical for both imploding and exploding shock 
waves. Our investigation reveals some unexpected puzzles. For example, for spherical explosions, we find a range of density profiles $5-\sqrt{3 / 4}<k<17 / 4$ for which current considerations allow for both first and second type solutions. Understanding which of these solutions will apply is left for later research. Fortunately, all our solutions are analytic. This is an advantage over the nonrelativistic case where the selfsimilar solutions are either implicit (first type) or numerical (second type). This property may facilitate the understanding of the interplay between first and second type solutions.

The plan of this article is as follows. We begin in Sec. II by writing down the one-dimensional flow equations in planar, cylindrical, and spherical symmetries. We then take the ultrarelativistic limit and assume self-similarity so the partial differential equations are reduced into ordinary differential equations. In Sec. III we explore the range in density power law index for which first type solutions, which obey global conservation law, apply and contain a finite energy. In Sec. IV we find second type solutions and explore the regimes where those are valid. The transition between first and second type solutions is explored in Sec. V.

\section{THE SELF-SIMILAR EQUATIONS IN ARBITRARY DIMENSIONS}

The flow equations are given by the conservation of energy, momentum, and particle number. Since in the relativistic case the particle number density does not play a role in the dynamics of the system, the conservation of particles equation is decoupled from the other two equations. Energy and momentum conservation yield

$$
\begin{gathered}
\frac{\partial}{\partial t} \gamma^{2}\left(e+\beta^{2} p\right)+\frac{1}{r^{\alpha}} \frac{\partial}{\partial r} r^{\alpha} \gamma^{2} \beta(e+p)=0, \\
\frac{\partial}{\partial t} \gamma^{2} \beta(e+p)+\frac{1}{r^{\alpha}} \frac{\partial}{\partial r} r^{\alpha} \gamma^{2} \beta^{2}(e+p)+\frac{\partial}{\partial r} p=0,
\end{gathered}
$$

and the particle conservation equation is

$$
\frac{\partial}{\partial t} \gamma n+\frac{1}{r^{\alpha}} \frac{\partial}{\partial r} r^{\alpha} \gamma \beta n=0
$$

where $\alpha=0,1$, and 2 for planar, cylindrical, and spherical symmetries, respectively. Here, $\gamma$ is the fluid Lorentz factor, $\beta$ is its velocity as a fraction of the speed of light, $n$ is the particle density, and $e$ is the rest frame energy density (see Ref. 12 for the derivation of similar equations).

We now assume that the flow has a characteristic Lorentz factor $\Gamma$ and a related characteristic position $R . R$ evolves with time in a way that describes motion with a Lorentz factor $\Gamma$. In the extreme relativistic case this implies $\dot{R}=\sqrt{1-1 / \Gamma^{2}} \approx 1-(1 / 2) \Gamma^{2}$. For the problem of interest here, it is natural to choose the position of the shock, and its Lorentz factor as $R$ and $\Gamma$. The characteristic length scale of the flow behind the shock must be $R / \Gamma^{2}$, so the similarity variable is

$$
\zeta=\frac{R-r}{R / \Gamma^{2}}=\Gamma^{2}(1-r / R) .
$$

BM doubted the use of Eq. (4) for $k>4$; however, Best and $\mathrm{Sari}^{8}$ demonstrated that this definition of the self-similar variable is applicable in general.

Note that in our notation $\dot{R}$ and $R-r$ are always positive. For converging solutions, this implies that $R$ is negative, growing toward zero. In our notation, therefore, converging solutions have a negative $\zeta$. This choice avoids the need to keep a positive or negative sign in the expression of $\dot{R}$ for imploding and exploding solutions.

The self-similar solution exhibits time dependent characteristic pressure and density, which we denote as $P(t)$ and $N(t)$. For consistency with the BM notation we define the self-similar variables as

$$
\begin{aligned}
\gamma^{2}(r, t) & =\frac{1}{2} \Gamma^{2}(t) g(\zeta), \\
p(r, t) & =P(t) f(\zeta), \\
n(r, t) & =N(t) h(\zeta) / g^{1 / 2}(\zeta),
\end{aligned}
$$

where $g, f$, and $h$ are functions of the self-similar variable $\zeta$ and respectively describe the spatial profiles of the Lorentz factor, pressure, and density.

In terms of the self-similar variables we have

$$
\frac{\partial}{\partial t}=\dot{\Gamma} \frac{\partial}{\partial \Gamma}+\left[2 \zeta \frac{\dot{\Gamma}}{\Gamma}+\left(\Gamma^{2}-1 / 2\right) \frac{r}{R^{2}}\right] \frac{\partial}{\partial \zeta}+\dot{P} \frac{\partial}{\partial P}+\dot{N} \frac{\partial}{\partial N}
$$

and

$$
\frac{\partial}{\partial r}=-\Gamma^{2} \frac{1}{R} \frac{\partial}{\partial \zeta} .
$$

The energy conservation equation now becomes

$$
0=2(-2 m+\alpha-k) g f-(1+2(m+1) \zeta)(g f)^{\prime}+f^{\prime} .
$$

As in the work of BM, we are motivated by this equation to define

$$
\chi=1+2(m+1) \zeta
$$

to obtain

$$
0=(-2 m+\alpha-k) g f-(m+1)\left[\chi \frac{d}{d \chi}(g f)-\frac{d}{d \chi} f\right] .
$$

Here we have used the notation $t \dot{\Gamma} / \Gamma=-m / 2, t \dot{P} / P=-m$ $-k$, and $t \dot{N} / N=-m / 2-k$. The relation between the time derivatives of $N, P$, and $\Gamma$ follows from the boundary conditions of strong relativistic shocks which imply $P \propto \Gamma N$. Since we set $\chi=1$ at the shock, those boundary conditions also imply $g(1)=f(1)=h(1)=1$ (see BM). Equation (12) shows that $m$ and $k$ must be constant to allow for a self-similar flow.

Repeating the same procedure for the momentum equation will again result in Eq. (12). This is because the energy and momentum equations are identical to the lowest order in $1 / \Gamma$ and our expansion discards any higher order terms. For 
this reason BM kept higher order terms. Instead, we find it simpler to use the difference equation between the energy and momentum conservation equations:

$$
\frac{\partial}{\partial t}\left(2+\frac{1}{\gamma^{2}}\right) p+\frac{1}{r^{\alpha}} \frac{\partial}{\partial r} r^{\alpha}\left(4-\frac{1}{\gamma^{2}}\right) p-2 \frac{\partial}{\partial r} p=0 .
$$

Keeping only lowest order terms is again sufficient here. With the self-similar variables Eq. (13) reads:

$$
\begin{aligned}
0= & (m+1)\left[-\chi g^{2} \frac{d f}{d \chi}+4\left(\frac{d f}{d \chi} g-\frac{d g}{d \chi} f\right)\right] \\
& +(-m-k+2 \alpha) g^{2} f .
\end{aligned}
$$

Similarly, for the particle conservation equation we have

$$
\begin{aligned}
\frac{d \log h}{d \chi}= & \left(-k g^{2}-m g^{2}+\alpha g^{2}-2(m+1) \frac{d g}{d \chi}\right) /\left(\chi g^{2}\right. \\
& -2 g) /(m+1) .
\end{aligned}
$$

We now solve Eqs. (12), (14), and (15) as a set of linear equations to get $d f / d \chi, d g / d \chi$, and $d h / d \chi$ and obtain:

$$
\begin{aligned}
& \frac{1}{g \chi} \frac{d \log g}{d \log \chi}=\frac{(7 m+3 k-2 \alpha)-(m+\alpha) g \chi}{(m+1)\left(g^{2} \chi^{2}-8 g \chi+4\right)}, \\
& \frac{1}{g \chi} \frac{d \log f}{d \log \chi}=\frac{4(2 m-\alpha+k)-(m+k-2 \alpha) g \chi}{(m+1)\left(g^{2} \chi^{2}-8 g \chi+4\right)},
\end{aligned}
$$

$$
\frac{1}{g \chi} \frac{d \log h}{d \log \chi}=\frac{2(9 m+5 k-4 \alpha)-2(5 m+4 k-3 \alpha) g \chi+(m+k-\alpha) g^{2} \chi^{2}}{(m+1)(2-g \chi)\left(g^{2} \chi^{2}-8 g \chi+4\right)} .
$$

For the case $\alpha=2$ these reduce to the equations given in BM.

\section{FIRST TYPE SOLUTIONS}

The parameter $m$ in the previous equations must be found in an independent way. As is well known in the nonrelativistic case, there are two types of self-similar solutions. In the first type, $m$ is found by global conservation laws. For arbitrary geometry, $\alpha$, in the ultrarelativistic regime this requirement reads

$$
E \sim \gamma^{2} R^{-k} R^{1+\alpha} \sim R^{-m-k+1+\alpha} \sim \text { const. }
$$

Therefore first type solutions have

$$
m=1+\alpha-k \text {. }
$$

Substituting this into the self-similar equations and using the boundary conditions $g(1)=f(1)=h(1)=1$ we obtain the very simple solution:

$$
\begin{aligned}
& g=\chi^{-1}, \\
& f=\chi^{(4 k-7-5 \alpha) /[3(2+\alpha-k)],}
\end{aligned}
$$

and

$$
h=\chi^{(2 k-3-2 \alpha) /(2+\alpha-k)} .
$$

This is a generalized form of the BM solution, and reduces to the BM solution in spherical geometry where $\alpha=2$.

First type solutions can be valid only if they contain a finite amount of energy. The energy in the solution is proportional to the integral

$$
\int f g d \chi \sim \chi^{(4 k-7-5 \alpha) /[3(2+\alpha-k)]} .
$$

This is finite if $1<\chi$ and $(4 k-7-5 \alpha) /[3(2+\alpha-k)]<0$ or if $0<\chi<1$ and $(4 k-7-5 \alpha) /[3(2+\alpha-k)]>0$. $1<\chi$ if $R(m+1)=R(2+\alpha-k)$ is positive. So the combination of the two previous possibilities requires $(4 k-7$ $-5 \alpha) R<0$. Therefore, explosions diverging to infinite distances $(R$ is positive) can be of first type if $k<(7+5 \alpha) / 4$. Converging solutions (where $R$ is negative approaching zero) are of first type only for $k>(7+5 \alpha) / 4$.

For $\chi<1$ these solutions are hollow and end at $\chi=0$. The Lorentz factor diverges at $\chi=0$, and it takes infinite time for a fluid element to arrive from the shock $(\chi=1)$ to $\chi=0$. Hollow solutions exist for diverging shocks in spherical symmetry with $4<k<17 / 4$ and for converging shocks in planar symmetry with $2>k>7 / 4$.

\section{SECOND TYPE SOLUTIONS}

Second type solutions do not obey global conservation laws. The true problem, therefore, cannot be completely described by a second type self-similar solution. Those describe only part of the flow, in some region of interest whereas other regions deviate from the solutions. In order not to influence the self-similar part, the non-self-similar parts must be separated from it by a sonic point, where the equations are singular. This requirement replaces the energy conservation as means of deducing the scaling of Lorentz factor with radius, i.e., finding $m$ (see Ref. 6 for a discussion of the nonrelativistic case and Ref. 8 for the relativistic case).

To find $m$ in second type solutions, we notice that the denominators of Eqs. (16)-(18) are independent of $\alpha$, therefore the sonic line has the same value when expressed in terms of $g \chi$. The dependence of $m$ on $k$ can be easily found, using the same method as in Best and Sari. ${ }^{8}$ Looking for roots of the denominator of our equations we find the sonic line to be 


$$
g \chi=4-2 \sqrt{3} \text {. }
$$

Although the fluid equations have other singular points, physical considerations (see Ref. 8) show that only the one given by (25) is the one we are looking for. This point separates the characteristic heading to the positive direction from the shock, which also goes in the positive direction per our definitions. The other singular points are $g \chi=2$, which represents a fluid element maintaining a fixed $\chi$, and $g \chi=4$ $+2 \sqrt{3}$, which corresponds to the negative characteristic hav- ing a fixed $\chi$. All those values are independent of the geometry, i.e., independent of the parameter $\alpha$.

Substituting Eq. (25) in the numerator of, for example, the equation of $g$ and demanding that it vanish, we obtain:

$$
m=-2 \alpha(5-3 \sqrt{3})+(3-2 \sqrt{3}) k .
$$

This reproduces the results of Best and Sari for $\alpha=2$. The hydrodynamic profiles can be found by substituting this value of $m$ in the equations for $g, f$, and $h$. The equations become very simple for this value of $m$ since by definition the denominator and the numerator have a common factor.

$$
g=K\left[\frac{1-\alpha}{-10 \alpha+6 \alpha \sqrt{3}+3 k-2 k \sqrt{3}+1} g \chi-2(2+\sqrt{3})\right]^{(3-2 \sqrt{3})(k-3 \alpha) /(\alpha-1)}
$$

where $K$ is an arbitrary constant. The boundary conditions $g(1)=1$ implies

$$
g=\left[\frac{g \chi(\alpha-1)+4 \alpha(\sqrt{3}-1)-2 k \sqrt{3}+4+2 \sqrt{3}}{(\alpha-1)+4 \alpha(\sqrt{3}-1)-2 k \sqrt{3}+4+2 \sqrt{3}}\right]^{(3-2 \sqrt{3})(k-3 \alpha) /(\alpha-1)} .
$$

For the pressure, we get

$$
\log f=K+\frac{(4-2 \sqrt{3})(k-3 \alpha)}{\alpha-1} \log [g \chi(\alpha-1)-4 \alpha+4 \alpha \sqrt{3}-2 k \sqrt{3}+4+2 \sqrt{3}]
$$

or, with the boundary conditions $f(1)=1$,

$$
f=\left[\frac{g \chi(\alpha-1)-4 \alpha+4 \alpha \sqrt{3}-2 k \sqrt{3}+4+2 \sqrt{3}}{\alpha-1-4 \alpha+4 \alpha \sqrt{3}-2 k \sqrt{3}+4+2 \sqrt{3}}\right]^{(4-2 \sqrt{3})(k-3 \alpha) /(\alpha-1)} .
$$

For the density we get

$$
\begin{aligned}
\log h= & K-\frac{(2 \sqrt{3}-3)(2 k-1+\alpha \sqrt{3}-3 \alpha)(k-3 \alpha)}{(-1-2 \alpha \sqrt{3}+k \sqrt{3}-\sqrt{3}+\alpha)(1-\alpha)} \log [(1-\alpha) g \chi+4 \alpha-4 \alpha \sqrt{3}+2 k \sqrt{3}-4-2 \sqrt{3}] \\
& -\frac{2 \alpha-k-\alpha \sqrt{3}}{-1-2 \alpha \sqrt{3}+k \sqrt{3}-\sqrt{3}+\alpha} \log (g \chi-2) .
\end{aligned}
$$

With the boundary conditions $h=1$ at $\chi=1$ we have

$$
\begin{aligned}
h= & {\left[\frac{(1-\alpha) g \chi+4 \alpha-4 \alpha \sqrt{3}+2 k \sqrt{3}-4-2 \sqrt{3}}{(1-\alpha)+4 \alpha-4 \alpha \sqrt{3}+2 k \sqrt{3}-4-2 \sqrt{3}}\right]^{[(2 \sqrt{3}-3)(2 k-1+\alpha \sqrt{3}-3 \alpha(k-3 \alpha)]][(\alpha-1-2 \alpha \sqrt{3}+k \sqrt{3}-\sqrt{3})(\alpha-1)]} } \\
& \times[2-g \chi]^{(-2 \alpha+k+\alpha \sqrt{3}) /(\alpha-1-2 \alpha \sqrt{3}+k \sqrt{3}-\sqrt{3})} .
\end{aligned}
$$

For cylindrical geometry, $\alpha=1$, the above-mentioned form is invalid and the solution (with the boundary condition of $g=1$ at $\chi=1$ ) reads

$$
\begin{aligned}
& \log g=\frac{g \chi-1}{2(2+\sqrt{3})}, \\
& \log f=\frac{3-2 \sqrt{3}}{3}(g \chi-1) .
\end{aligned}
$$

The condition we used to demand a smooth transition through the sonic point, which led to Eq. (26), is necessary but not sufficient. We still need to verify that the solution passes through the sonic point in the relevant range of the independent variable $\chi$. We therefore substitute $g \chi=4-2 \sqrt{3}$ in the expression for $g$ and find the value $\chi_{\text {sonic }}$. This is displayed in Fig. 1 for the three possible values of $\alpha$. We demand that $\chi_{\text {sonic }}$ is within the range of values that $\chi$ takes, i.e., we require $\chi_{\text {sonic }}>1$ for diverging solution with $m>-1$ or converging solutions with $m<-1$, and we require $\chi_{\text {sonic }}$ $<1$ for diverging solutions with $m<-1$ or converging solu- 

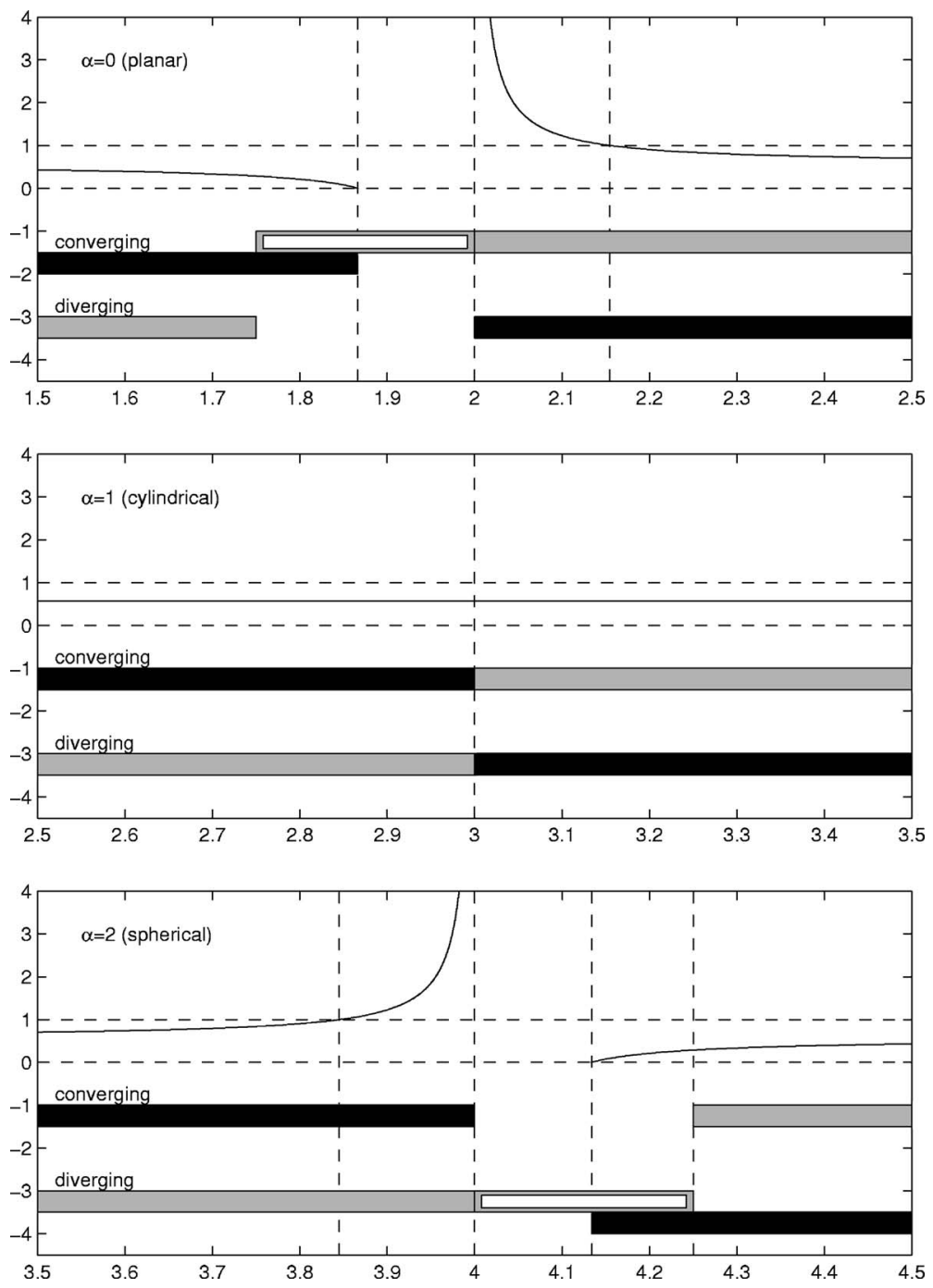

FIG. 1. Schematic description of the possible solutions for one-dimensional strong relativistic explosions. The three panels are for planar (top), cylindrical (middle), and spherical geometries (bottom). The $x$ axis is the density index $k\left(\rho \propto r^{-k}\right)$. Gray stripes indicate the region where first type solutions exist $(k$ $<4$ in the spherical case), and black stripes show where a second type solution exists. Hollow first type solutions, in which all the shocked fluid is confined between the shock and some internal surface are indicated by a hollow grey stripe. The $y$ axis of gray and black stripes is arbitrary. The solid line is the position of the sonic point, $\chi_{\text {sonic }}$.

tions with $m>-1$. The results regarding the validity range of type-II solutions are given in Table I and shown schematically in Fig. 1.

A necessary condition for a second type solution to be correct, is that its energy decrease with time, that is, $m>1$ $+\alpha-k$ for diverging solutions and the opposite for converging. We demonstrate this now for diverging solutions:

$$
m_{I I}=-2 \alpha(5-3 \sqrt{3})+(3-2 \sqrt{3}) k>1+\alpha-k=m_{I}
$$

or

$$
k>(1+11 \alpha-6 \alpha \sqrt{3}) /(4-2 \sqrt{3}) .
$$

This gives $k>1+\sqrt{3 / 4}$ for planar symmetry, $k>3$ for cylindrical symmetry and $k>5-\sqrt{3 / 4}$ for the spherical case. This

TABLE I. The validity range of second type solutions. The choice of $m$ according to Eq. (26) guarantees that if a solution passes through a sonic point, the solution is smooth there. The conditions listed verify that the solution does in fact pass through the sonic line within the physically relevant range of $\chi$.

\begin{tabular}{cccccc}
\hline \hline \multirow{2}{*}{ Symmetry } & \multicolumn{2}{c}{ Diverging solutions $(R \rightarrow \infty)$} & & \multicolumn{2}{c}{ Converging solutions $(R \rightarrow 0)$} \\
\cline { 2 - 3 } \cline { 5 - 6 } & Type-I & Type-II & & Type-I & Type-II \\
\hline Planar & $k<7 / 4$ & $k>2$ & & $k>7 / 4$ & $k<1+\sqrt{3 / 4} \cong 1.87$ \\
Cylindrical & $k<3$ & $k>3$ & & $k>3$ & $k<3$ \\
Spherical & $k<17 / 4$ & $k>5-\sqrt{3 / 4} \cong 4.13$ & & $k>17 / 4$ & $k<4$ \\
\hline \hline
\end{tabular}



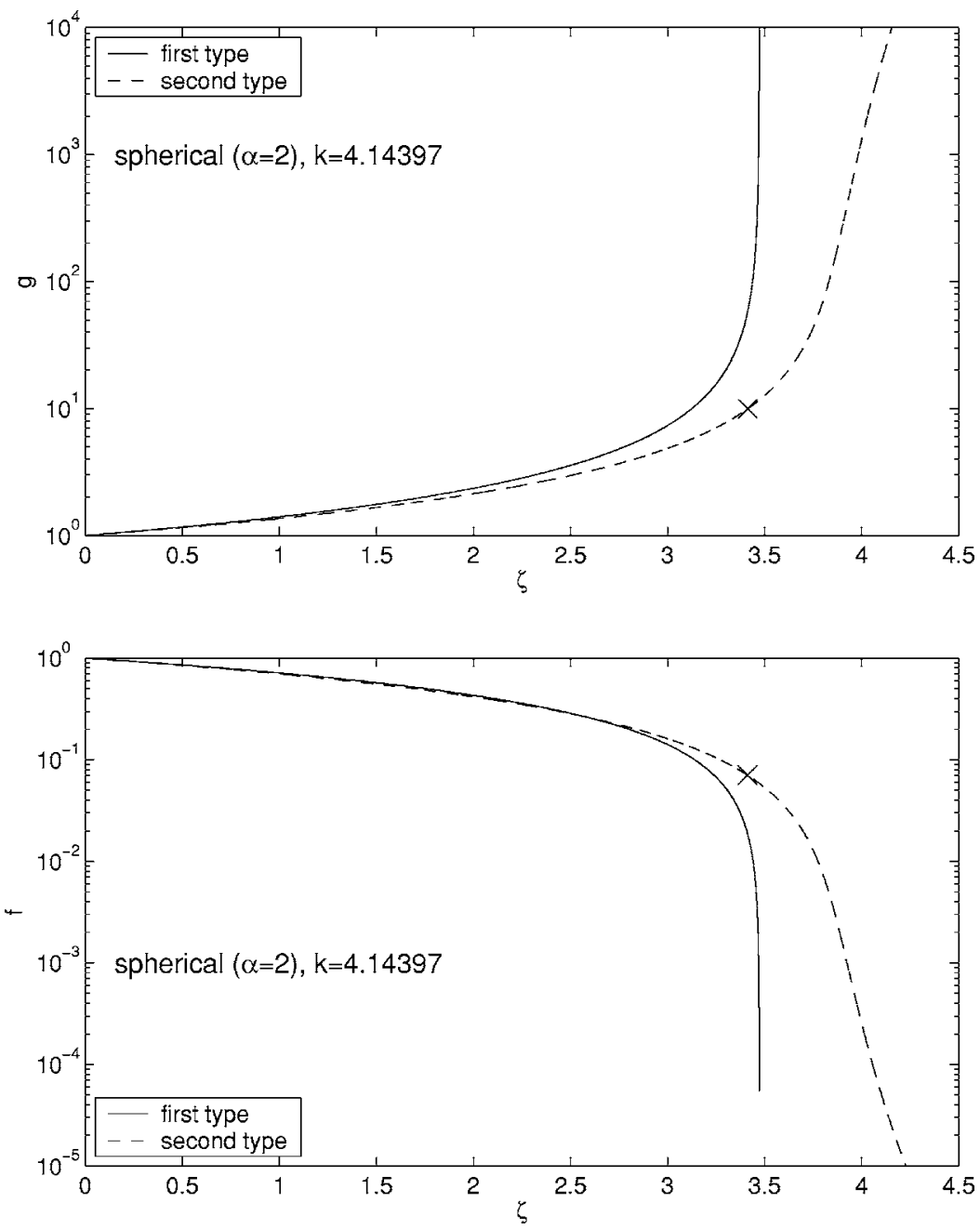

FIG. 2. First and second type solutions for $k=5-\sqrt{3 / 4}+0.01$ plotted as a function of $\zeta$. The top plot shows the Lorentz factor squared profile, $g$, and the bottom plot the pressure profile, $f$. The two solutions are very similar close to the shock $(\zeta=0)$. The type-I solution is hollow, so the pressure drops to zero and $\gamma$ diverges at some finite distance from the shock $\zeta=-1 /(2 m+2) \cong 2+\sqrt{3}$. The second type solution continues smoothly but does show a relatively sharp drop around the same distance.

is completely consistent with the above-mentioned requirements. Indeed the requirement of decreasing energy follows from the condition that the solution passes through the sonic point: if the flow behind the sonic point cannot influence the flow ahead of the sonic point, energy cannot flow from behind the sonic point.

\section{THE TYPE-I TO TYPE-II TRANSITION}

Note, that when $m_{\mathrm{I}}=m_{\mathrm{II}}$ the two solutions listed previously are the same, despite their seemingly different expressions. Clearly this must be the case since for a given $m$ the solution to the self-similar equations with the shock boundary conditions is unique. Nevertheless, it is instructive to verify this from the two explicit expressions given in the previous sections [e.g., Eqs. (21) and (28)]. Let us consider the spherical case where $m_{\mathrm{I}}=m_{\mathrm{II}}$ at $k=5-\sqrt{3 / 4}$, and start by examining the second type solution. For $k \approx 5-\sqrt{3 / 4}$, the denominator of Eq. (28) is close to zero. This means than $g \chi$ has to be close to unity to make the numerator close to zero as well. This agrees with the expression for $g$ in the first type solution $g=\chi^{-1}$.
Where is the sonic point in type-II solutions for $k$ close to $k=5-\sqrt{3 / 4}$ ? Expanding Eq. (28) we obtain

$$
g_{\text {sonic }} \rightarrow\left[\frac{1-\sqrt{3 / 4}}{k-(5-\sqrt{3 / 4})}\right]^{\sqrt{3 / 4}}
$$

So that $g_{\text {sonic }}$ diverges when $k$ is close to $k=5-\sqrt{3 / 4}$. Since at the sonic point $g_{\text {sonic }} \chi_{\text {sonic }}=4-2 \sqrt{3}$ is finite, $\chi_{\text {sonic }}$ approaches zero. This is very similar to the behavior of type-I solutions as those end at $\chi=0$ with divergence of $g$. Nevertheless, type-II solutions continue past $\chi=0$ into negative $\chi$ and they are not hollow. A plot of first and second type solutions near the transition is given in Fig. 2.

An unfortunate property of the solutions around $k=4$ (for the diverging case in spherical geometry) is that the profile approaches nonrelativistic temperatures very quickly as a function of the distance from the shock. We define nonrelativistic temperatures to occur where $p / n<1$. We have 


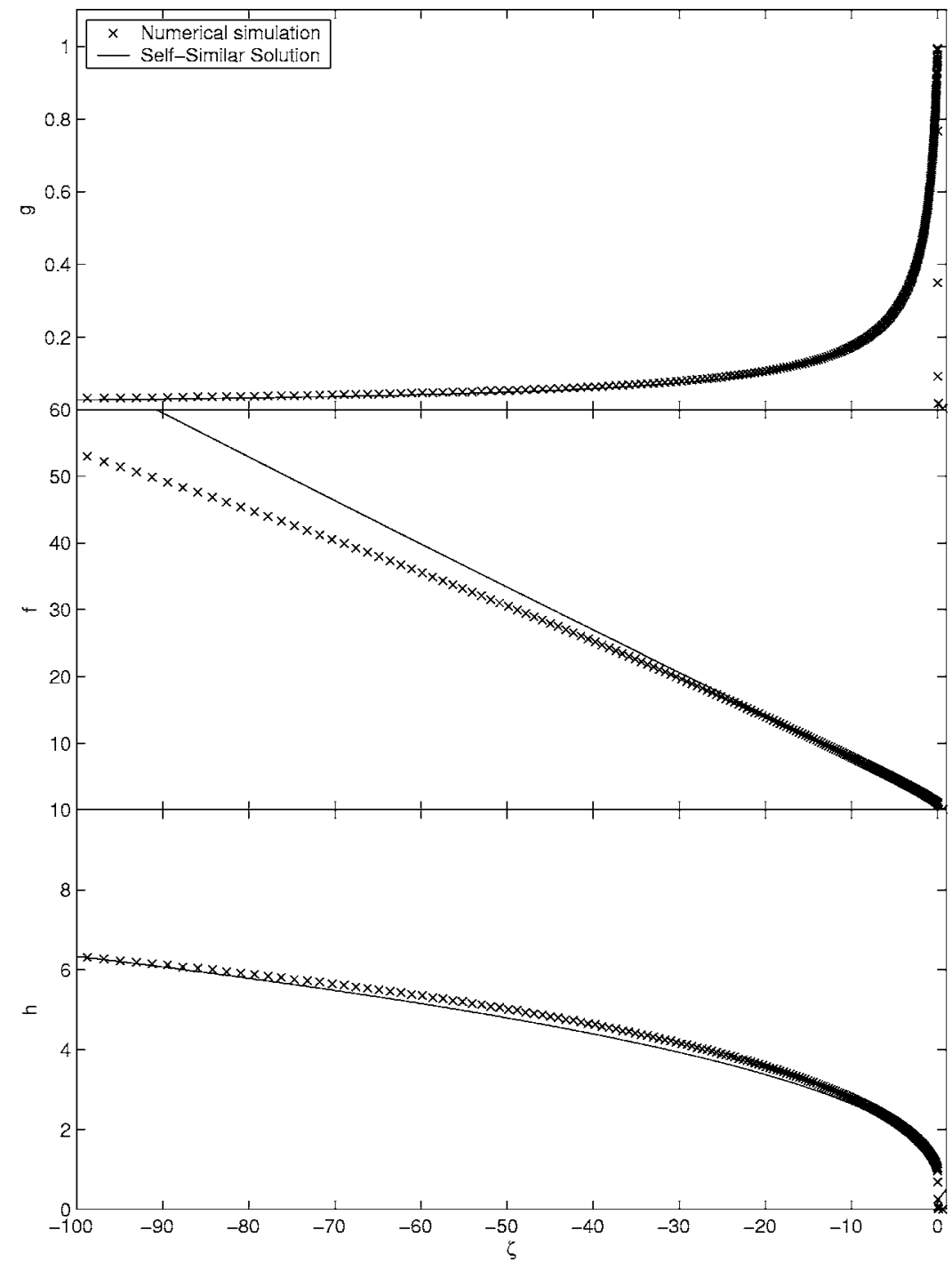

FIG. 3. Comparison of numerical integration of the time dependent hydrodynamical equations and the prediction of the self-similar solution. Plotted, top to bottom, are the Lorentz factor, the pressure and density relative to that just behind the shock, for an imploding spherical shockwave into matter of constant density: $\alpha=2$ and $k=0$.

$$
\begin{aligned}
\frac{p}{n} \sim \Gamma \frac{f \sqrt{g}}{h} & \sim \Gamma(1+2(4-k) \zeta)^{(k+4) /[6(k-4)]} \\
& \sim \gamma \exp (-8 \zeta / 3) .
\end{aligned}
$$

So the temperature becomes nonrelativistic at

$$
\zeta_{N R} \approx \frac{3}{8} \ln \Gamma
$$

This is just a few times $R / \Gamma^{2}$ behind the shock, even for quite large $\Gamma$. For $k \approx 4$ the solution extends many times $R / \gamma^{2}$ behind the shock. We therefore expect that for moderate values of $\Gamma$, the ultra relativistic self-similar solution gives an approximate rather than an accurate description of the flow.

Clearly it is of importance to understand which of the two solutions apply. In principle, one can attempt this with numerical simulations. However, the problem of nonrelativ- istic temperatures noted previously makes numerical investigation difficult as it requires extremely large Lorentz factors which are hard to achieve.

\section{DISCUSSION}

We have explored the possible ultrarelativistic selfsimilar solutions in planar, cylindrical, and spherical geometries, containing diverging or converging shocks, and allowing for a general power law density profile $\rho \propto r^{-k}$. Relativistic implosions in all three geometries and with a general power law density profile are treated here for the first time (imploding relativistic shock waves for spherical geometry with constant density where treated numerically by Ref. 11). The hydrodynamic profiles of such a self-similar implosion are compared with numerical integration of the time dependent equations in Fig. 3.

We show that as a function of the density power law index $k$, there is a sequence of self-similar solutions. In the 
explosive scenarios, the sequence is from first type solutions to hollow first type solutions and then second type solutions as $k$ increases. The sequence is reversed for imploding solutions. Those are of second type for low $k$ and first type for large $k$. In both cases, explosions and implosions, the solution is of second type if the density ahead of the shock decreases sufficiently fast and of first type if it increases sufficiently fast. The physical interpretation of this is clear. A shock wave that propagates into a density profile that decreases sufficiently fast will accelerate and "run away" from the fluid behind it. The shock becomes "causally disconnected" from the downstream fluid, i.e., a second type solution. On the other hand, if the shock wave does not accelerate enough, sound waves from behind it will be able to catch up and the whole flow is causally connected, i.e., a first type solution. In some of the geometries (converging planar case and diverging spherical case) first type solutions become hollow before they turn into second type solutions. This sequence of self-similar solutions and its physical origin apply to the nonrelativistic case as well.

Our analysis raises some interesting riddles. In some cases (planar diverging shocks or spherical converging shocks) this sequence has a gap between the first and second type solutions. In other cases (planar converging shocks and spherical diverging shocks) there seems to be an overlap in the sequence between first and second type solutions: current considerations allow for the existence of both self-similar solutions for the same value of $k$.

Recently, Gruzinov ${ }^{13}$ suggested a solution in the gap region in the nonrelativistic case which he called a "third type" self-similar solution. In his third type solution, the infinite mass located at the origin acts like a piston moving at constant velocity. Preliminary investigation seems to support a relativistic analog of Gruzinov's analysis. In the relativistic case, however, the solutions are more interesting since the piston with large inertia does not move at a constant speed but accelerates. Further research is needed to establish this connection.

\section{ACKNOWLEDGMENTS}

This research was partially supported by a NASA ATP grant. RS is a Packard Fellow and an Alfred P. Sloan Research Fellow.

${ }^{1}$ G. I. Taylor, "The formation of a blast wave by a very intense explosion 1. Theoretical discussion,” Proc. R. Soc. London, Ser. A 201, 159 (1950).

${ }^{2}$ J. von Neumann, Blast Waves (Los Alamos Science Laboratory Technical Series, Los Alamos, NM, 1947), Vol. 7.

${ }^{3}$ L. Sedov, Similarity and Dimensional Methods in Mechanics (Academic, New York, 1969), Chap. IV.

${ }^{4}$ G. Guderley, "Starke kugelige und zylindrische verdichtungsstobe in der nahe des kugelsmittelpunkts bzw des zylinderachse," Luftfahrtforschung 19, 302 (1942).

${ }^{5}$ Ya. B. Zel'dovich and Yu. P. Raizer, Physics of Shock Waves and HighTemperature Phenomena (Academic, New York, 1966), Chap. IV.

${ }^{6}$ E. Waxman and D. Shvarts, "Second-type self-similar solutions to the strong explosion problem," Phys. Fluids A 5, 1035 (1993).

${ }^{7}$ R. D. Blandford and C. F. McKee, "Fluid dynamics of relativistic blast waves," Phys. Fluids 19, 1130 (1976).

${ }^{8}$ P. Best and R. Sari, "Second-type self-similar solutions to the ultrarelativistic strong explosion problem," Phys. Fluids 12, 3029 (2000).

${ }^{9}$ R. Perna and M. Vietri, "self-similar solutions for the propagation of a relativistic shock in an exponential atmosphere," Astrophys. J. Lett. 569, L47 (2002).

${ }^{10}$ Yu. P. Raizer, "Propagation of a shock wave in an inhomogeneous atmosphere in the direction of decreasing density," Zh. Prikl. Mat. Tekh. Fiz. 4, 49 (1964).

${ }^{11}$ J. C. Hidalgo and S. Mendoza, "Self-similar imploding relativistic shock waves," Phys. Fluids 17, 96101 (2005).

${ }^{12}$ L. D. Landau and E. M. Lifschitz, Fluid Mechanics, 2nd ed. (Pergamon, New York, 1987).

${ }^{13}$ A. Gruzinov, preprint astro-ph/0303242. 